\title{
Hot or Cold ? Feasibility, Safety and Outcome after Maze-like Radiofrequency guided versus Cryoballoon guided LAA Isolation
}

\author{
Shota Tohoku ${ }^{1}$, Shaojie Chen ${ }^{2}$, Stefano Bordignon ${ }^{1}$, Fabrizio Bologna ${ }^{1}$, Simone Zanchi ${ }^{1}$, \\ Lorenzo Bianchini $^{1}$, Felix Operhalski ${ }^{3}$, KR Julian Chun ${ }^{1}$, and Boris Schmidt ${ }^{1}$ \\ ${ }^{1}$ Cardioangiologisches Centrum Bethanien \\ ${ }^{2}$ Agaplesion Markus Krankenhaus \\ ${ }^{3}$ Universitätsklinikum Frankfurt
}

April 26, 2021

\begin{abstract}
Backgrounds: Left atrial appendage (LAA) isolation (LAAI) has been described as an adjunctive ablation strategy for patients with recurrent atrial fibrillation (AF). Objectives: We compared the clinical impact of persistent LAAI durability between radiofrequency catheter $(\mathrm{RF})$-guided wide-area LAAI and cryoballoon (CB)-guided ostial LAAI. Methods: Consecutive patients who underwent RF- or CB-guided LAAI were retrospectively analyzed. RF-guided LAAI was performed by combining anterior, roof and mitral isthmus linear ablation. CB-guided LAAI was performed by LAA ostial ablation. After LAAI, patients underwent invasive re-mapping study. LAA closure was performed if persistent durability was confirmed. Procedural data, LAAI durability and ATa recurrence were assessed. Results: A total of 260 patients (RF:n=201, CB:n=59) undergoing LAAI were identified out of $7630 \mathrm{AF}$ ablation procedures. Acute rate of procedural LAAI was significantly higher in CB group (RF: $82.6 \%$ vs. $\mathrm{CB}: 94.9 \%, \mathrm{P}=0.02$ ) and associated with a lower rate of pericardial effusion (RF: $7.5 \%$ vs. $\mathrm{CB}: 0 \%, \mathrm{P}=0.03$ ). Six-week durable LAAI was similar between two groups (RF: $78.1 \%$ vs. CB: $66.0 \%, \mathrm{P}=0.103$ ). One-year freedom from ATa recurrence was higher in the patients with durable LAAI after RF-guided wide-area LAAI irrespective of arrhythmia types (overall; RF:76.3\% vs. CB:56.7\%, $\mathrm{P}=0.0017$, only $\mathrm{AF}$; RF:81.3\% vs. CB:57.5\%, $\mathrm{P}=0.0013$, respectively). Multivariate analysis revealed that RF-guided LAAI was a predictor of freedom from ATa recurrence (HR: 0.41, 95\%CI: 0.221-0.766, P=0.0056). Conclusions: Acute LAAI can be more readily and safely achieved by CB-guided ostial ablation. In patients with confirmed LAAI, however, the freedom from ATa recurrence was higher after a RF-guided wide-area isolation.
\end{abstract}

\section{Hosted file}

black JCE2021 MS-LAA isolation_.pdf available at https://authorea.com/users/336771/articles/ 519508-hot-or-cold-feasibility-safety-and-outcome-after-maze-like-radiofrequency-guidedversus-cryoballoon-guided-laa-isolation 